\title{
The Tumor Vascular Endothelium as Decision Maker in Cancer Therapy
}

\author{
Diana Klein * \\ Institute of Cell Biology (Cancer Research), University Hospital, University of Duisburg-Essen, Essen, Germany
}

Genetic and pathophysiologic criteria prearrange the uncontrolled growth of neoplastic cells that in turn initiates new vessel formation, which is prerequisite for further tumor growth and progression. This first endothelial lining is patchy, disordered in structure and thus, angiogenic tumor vessels were proven to be functionally inferior. As a result, tumors were characterized by areas with an apparent oversupply in addition to areas with an undersupply of vessels, which complicates an efficient administration of intravenous drugs in cancer therapy and might even lower the response e.g. of radiotherapy (RT) because of the inefficient oxygen supply. In addition to the vascular dysfunction, tumor blood vessels contribute to the tumor escape from immunity by the lack of response to inflammatory activation (endothelial anergy) and by repression of leukocyte adhesion molecule expression. However, tumor vessels can remodel by the association with and integration of pericytes and smooth muscle cells which stabilize these immature vessels resulting in normalization of the vascular structures. This normalization of the tumor vascular bed could improve the efficiency of previously established therapeutic approaches, such as chemo- or radiotherapy by a more homogenous drug and oxygen distribution, and/or by overcoming endothelial anergy. This review highlights the current investigations that take advantage of a proper vascular function for improving cancer therapy with a special focus on the endothelial-immune system interplay.

Keywords: neovascularization, angiogenesis, radiotherapy, anti-angiogenic therapy, vascular stabilization, immune escape

\section{INTRODUCTION}

New vessel formation is a hallmark of tumor growth and progression (1-3). Once a critical tumor mass (of approximately $1-2 \mathrm{~mm}^{3}$ ) has formed, the metabolic demands of the growing cancer cells together with the diffusion limits of nutrients and oxygen foster the generation of a tumorassociated neovasculature (4). Known as the angiogenic switch, this process is regulated directly and indirectly by the tumor using a variety of pro- and anti-angiogenic signaling molecules, including vascular endothelial growth factor (VEGF), platelet-derived growth factor (PDGF), angiopoietins and thrombospondins, among others $(5,6)$.

In contrast to the normal, usually quiescent vasculature, tumor blood vessels were proved to be functionally abnormal because of their immature phenotype: the endothelial lining is patchy, the basement membrane is defective or discontinuous and respective vessel walls lack the mural elements (smooth muscle cells and pericytes); so they cannot actively respond to physiologic stimuli (Figure 1) $(7,8)$. Thus, there is relative imbalance between tumor tissue and the formation of adequate vascular structures, which finally results in tumor areas with an apparent oversupply in addition to areas with an undersupply of vessels. This complicates not only the efficient 


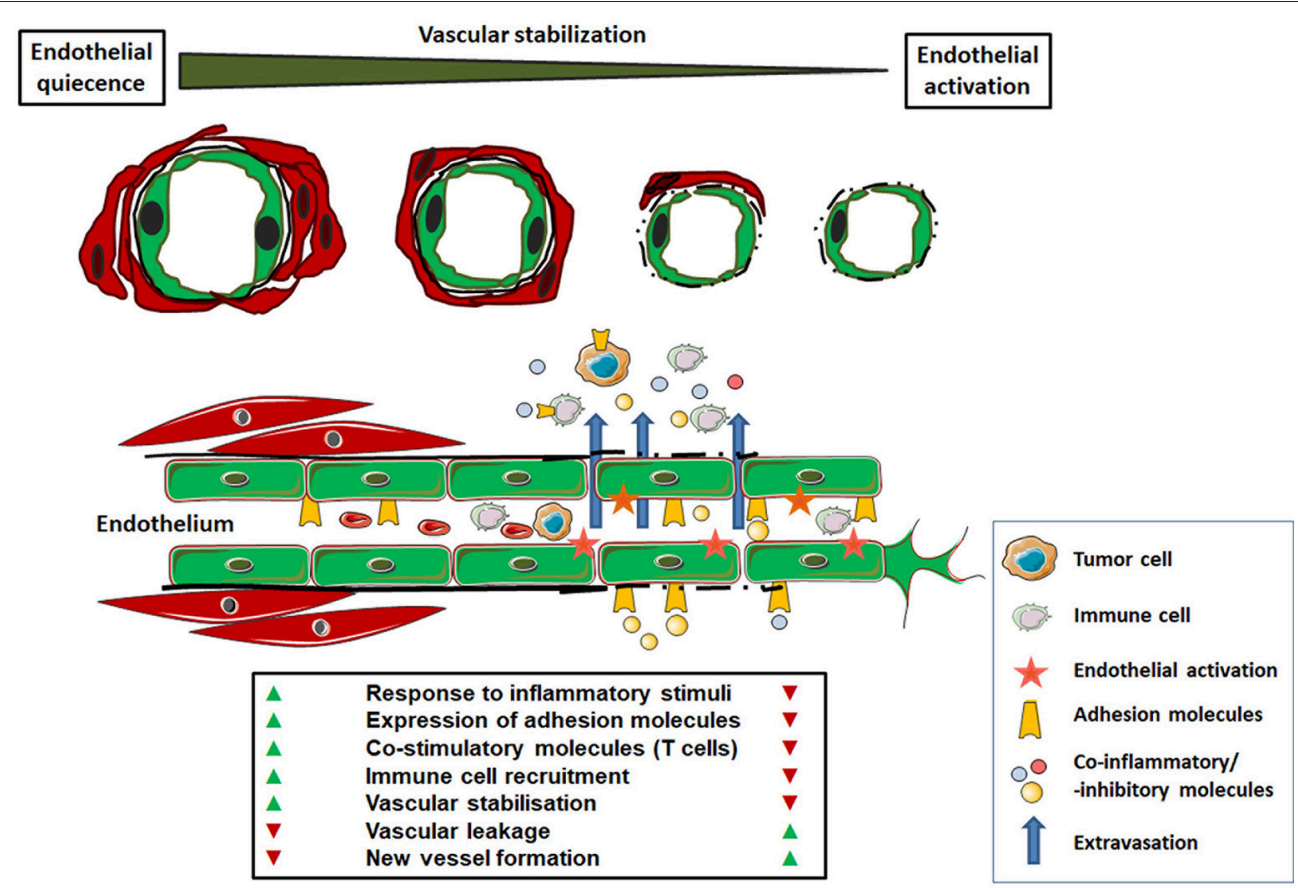

FIGURE 1 | Functional characteristics of normal versus tumor-associated endothelium. In the healthy state, mature endothelial cells (shown as green cells) are characterized by quiescence. A regular blood flow and pressure is achieved by vascular stabilization, which is the association and integration of vascular mural cells (smooth muscle cells and pericytes; shown as red cells). Thus, the normal endothelium provides an efficient barrier to liquids or cell extravasation. Upon activation, e.g., in response to inflammatory signals, normal endothelial cells can up-regulate cell adhesion molecules (selectins and integrins) for the capture, rolling and arresting of circulating immune cells prior tissue extravasation. The anergic tumor endothelium lacks that response to inflammatory stimuli. In response to tumor-secreted angiogenic factors the endothelium becomes activated. This activated and/or "angiogenic" endothelium phenotype is characterized by a missing or defective basement membrane and structural instability (lack of vascular mural cells), which leads to increased vascular leakage. In addition, these newly formed and functional abnormal blood vessels are chaotically organized which, together with endothelial anergy, limits the effective immune cell distribution and tissue infiltration. The altered expression of co-stimulatory and-inhibitory molecules with the potential to block anti-tumor immune cells further contributes to an immunosuppressive microenvironment within the tumor.

distribution of nutritions and oxygen but also the effective administration of cancer therapeutics. Even at the molecular level, i.e., regarding the expression of important signaling molecules, receptors or cell adhesion molecules in the tumor vascular bed, there is an imbalanced state between pro- and anti-oxidants, -inflammatory molecules, and -coagulation signals (9-11). As a result, tumor endothelial cells bear immuneregulatory properties: alterations in the immune cell attraction and activation, as well as in the expression of co-stimulatory and -inhibitory molecules can promote immune tolerance and thus generate an immune-privileged tumor microenvironment (12-14).

However the newly formed tumor vessels can remodel in terms of vascular maturation within the course of tumor progression $(7,10,15,16)$. Herein, a partial stabilization, which is achieved by the association and integration of vascular mural cells occurs particularly in the central areas of the tumors, which is associated with a significant reduction of vascular densities and augmented necrosis in these tumor regions (Figure 2) (7, $16,17)$. The process of vascular remodeling within a tumor is influenced by the cancer therapy. Especially in anti-angiogenic therapy, angiogenesis inhibitors foster vascular stabilization and a partial normalization of the tumor vascular bed, which is supposed to improve the efficiency of the previously established therapeutic approaches, such as chemo-, radio-, and/or immunotherapy (18-20). This review highlights the central role played by the tumor vascular endothelium for cancer therapy and summarizes the current strategies that take advantage of a proper vascular function for overcoming anti-tumor immunity and thus improving immunotherapy.

\section{ENDOTHELIAL ACTIVATION AND DYSFUNCTION}

One important physiological function of normal endothelial cells is quiescence of the inflammatory response and thus, participation in immune surveillance $(21,22)$. Quiescent endothelial cells fail to provide the requisite signals for leukocyte recruitment; but the cells can be activated to express adhesion molecules and to release chemokines that promote capture and transmigration of blood leukocytes into tissues. Endothelial cell activation can typically induced by multiple factors, including circulating inflammatory cytokines, such as tumor necrosis factors (TNF) and interleukins (IL), reactive oxygen species, oxidized low density lipoprotein, autoantibodies and traditional 


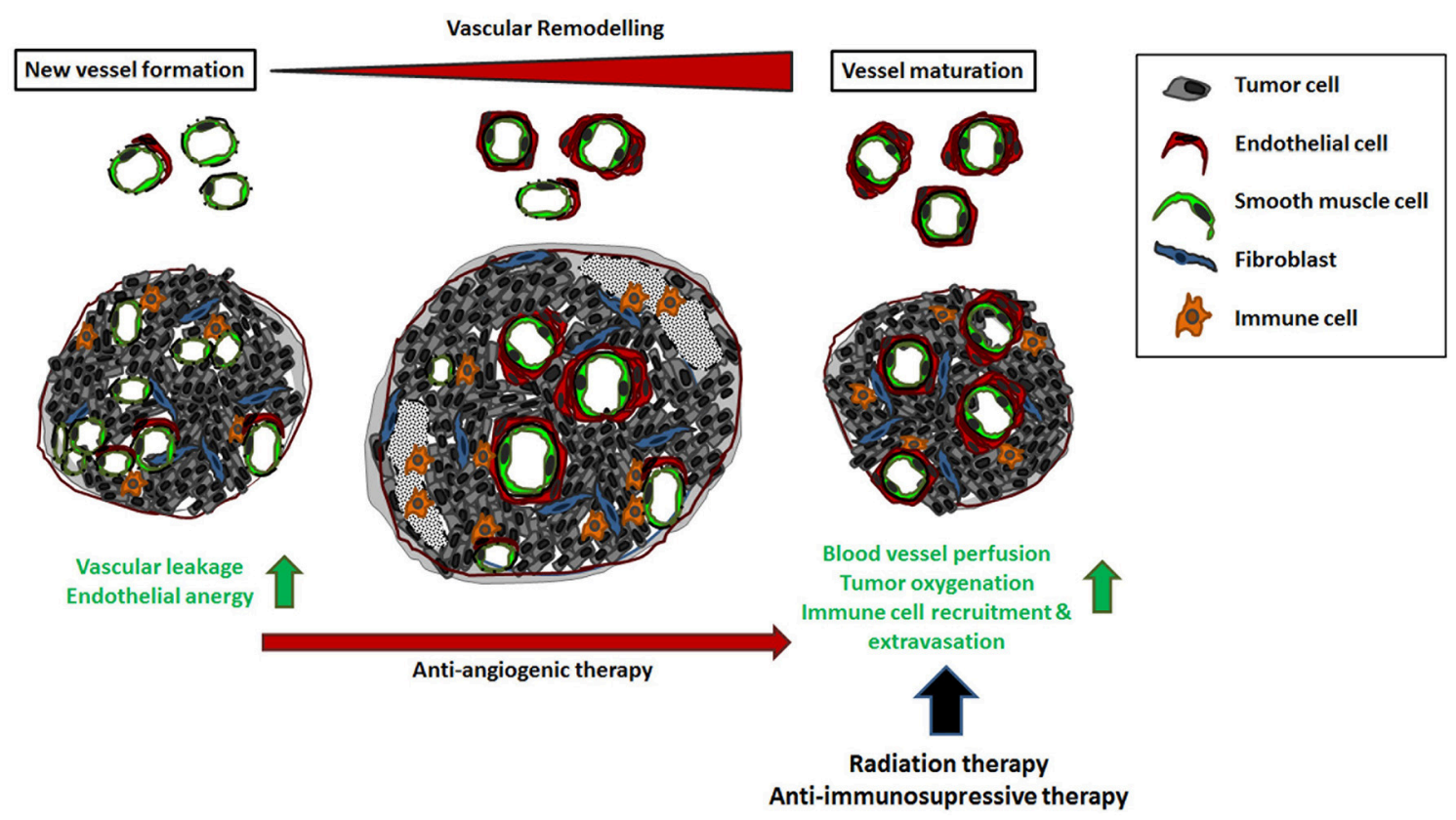

FIGURE 2 | The impact of vascular remodeling for cancer therapy. Tumor neovascularization supplies a high dense network of chaotically organized, immature and unstable vessels. Vascular dysfunction as well as the unresponsiveness to inflammatory stimuli results in an uneven blood flow and pressure as well as an inefficient distribution of blood stream components, including circulating immune cells. This complicates the effective administration of cancer therapeutics. In the course of tumor progression, these angiogenic vessels can mature by the association of vascular mural cells (vascular remodeling) that stabilizes the immature vessels resulting in normalization of the vascular structures. Vascular remodeling is dynamic and strictly regulated process; an ordered remodeling seems to be critical for proper vascular development, maintenance and stability of the vessel wall. The process of vascular stabilization is accelerated in cancer therapy when anti-angiogenic agents were applied. As a result, blood vessel perfusion and thus oxygenation as well as the efficient distribution of applied drugs are improved. In addition, vascular maturation and normalization restores the potential of the tumor endothelium to recruit and direct circulating immune cells to the tumor tissue.

risk factors directly and indirectly activate endothelial cells (21). The term activated endothelium implies a change in endothelial cell morphology (23). Endothelial activation was further specified as a change in surface molecules and in endothelial cell functions in response to cytokine treatment, and it was emphasized that these changes does not represent endothelial cell injury or dysfunction $(24,25)$. Components of endothelial cell activation are upregulation of surface antigens (e.g., HLA molecules) and leucocyte adhesion molecules (e.g., E-selectin, ICAM-1/2, and VCAM-1), pro-thrombotic endothelial cell changes (e.g., loss of the surface anticoagulant molecules thrombomodulin and heparan sulfate), cytokine production (e.g., IL6, IL8, MCP1), and changes in the vascular tone (e.g., loss of vascular integrity, expression of vasodilators, and NO). These components mutually interact in causing local inflammation (25). Endothelial activation also leads to an increase in angiopoietin-2, which is known to destabilize barrier function and promote inflammation (26). The recruited and extravasated immune cells appear then in vicinity of the activated endothelial cells, and can further become activated (23). Importantly, the phenotype of activated endothelial cell is reversible and can return to the quiescent, non-activated phenotype when the activating factors were removed (27-30). Prolonged activation of the endothelium can be associated with the loss of microvascular barrier integrity and subsequent vascular injury or progress to endothelial cell apoptosis (31).

\section{THE TUMOR ENDOTHELIUM}

Phenotypic differences at the molecular and functional levels have been identified for tumor and normal endothelial cells (32). Tumor secreted growth factors, and in particular VEGF, are the principal drivers of most the fundamental morphogenetic events involved in the induction of tumor vascularization including activation of the hitherto quiescent endothelium in terms of stimulating endothelial cell proliferation and migration (33). Many tumor types are characterized by a VEGF upregulation. Tumor hypoxia can also foster increased VEGF expression levels, which in turn perpetuates angiogenic processes (34). Tumor endothelial cell are very heterogeneous and thus vascular function of respective tumor blood vessels vary depending on the type of tumor and progression stage $(35,36)$. The newly formed blood vessels of tumors as well as of metastatic tumors are more immature with fewer pericytes. In general, tumor endothelial cells are characterized by a proangiogenic phenotype, with the upregulation of several angiogenesis-related genes, such as VEGFR1/R2 and matrix metalloproteinases (MMPs) to modulate the basement membrane and degrade the extracellular matrix allowing endothelial cell migration. The resulting tumor vascular bed is disorganized, tortuous, and the leaky phenotype of angiogenic tumor blood vessels that is accompanied by an irregular blood and heterogeneous permeability limits for the efficient distribution of blood components within the 
tumor mass. Further on, the structural abnormalities like poorly interconnected endothelial cells, no regular associated mural cells, and abundance of vesiculo-vacuolar organelles contribute to the leaky, hyper-permeable phenotype, finally causing extravasation of intravascular fluids and plasma proteins $(37,38)$. Therefore, an markedly increase in the intra-tumor fluid pressure throughout the tumor is observed, while normal pressure values were found in the tumor's periphery or in the surrounding tissue $(39,40)$. The high tissue pressure within the tumor, together with mechanical stress from the proliferating cancer cells and the extra mass of generated matrix, is able to collapse tumor vessels, that means closing their lumen through compressive forces, leading to the collapse of the blood vessels and finally resulting in hypoxia $(32,38,41)$. The compromised blood flow in tumor blood vessels further decreases oxygen and nutrient supply, causing physiological stress to the tumor. The physiological microenvironments of many macroscopic tumors were therefore characterized by high interstitial fluid pressure (interstitial hypertension), which besides nutrient deprivation and hypoxia in turn was associated with malignant progression, development of metastatic disease and a poor disease-free survival in a large number of cancer types (42-44).

Angiogenic growth factors were further shown to suppress the expression of adhesion molecules involved in leukocyte binding (e.g., ICAM-1/2, VCAM-1, E-selectin and CD34) in tumor endothelial cells, which then causes the unresponsiveness of tumor endothelial cells to inflammatory signals, a phenomenon called endothelial cell anergy that causes lymphocyte tolerance (45-48). Hence, the interaction of leukocytes with the endothelial cells lining the vessels is reduced, and thus intra-tumoral recruitment of effector $\mathrm{T}$-cells, either induced or adoptively transferred, is impaired and subsequently fail to exert the antitumor effects necessary to eradicate the tumor $(49,50)$. This is one of the mechanisms tumors have developed to escape the immune surveillance (51). Concerning the mechanism, angiogenic growth factor like VEGF and bFGF inhibited the TNF-mediated activation of NF-KB. In addition, bFGF induced hyperphosphorylation of p38 MAPK on endothelial cells $(52,53)$. Promoter histone modifications were further shown to mediate tumor endothelial cell anergy, as adhesion molecule expression was shown to be epigenetically repressed in tumor endothelial cells, and that DNA methyltransferase and histone deacetylase inhibitors which have angiostatic activity could re-induce expression of the ICAM-1 gene by reversal of histone modifications in the ICAM-1 promoter, thereby restoring leukocyte-vessel wall interactions and leukocyte infiltration (51).

\section{TUMOR ENDOTHELIUM-MEDIATED REGULATION OF THE IMMUNE RESPONSE: CLINICAL IMPLICATIONS FOR TARGETING THE TUMOR VASCULATURE TO IMPROVE IMMUNOTHERAPY}

A functional vascular network is prerequisite not only for nutrients or oxygen supply but also for the immune cells to enter the tissues. The functional and structural abnormalities of tumor blood vessels together with the unresponsiveness of the endothelium to inflammatory stimuli caused by proangiogenic factors decrease the recruitment of immune effector cells into the tumor, thus limiting the effectiveness of cancer immunotherapies $(54,55)$. Given that the abnormal tumor vasculature contributes to the immune-suppressive tumor microenvironment, processes of vascular normalization in terms of vessel maturation were supposed to potentiate cancer immunotherapy by promoting immune cell infiltration into tumors and reducing the immune suppression within the tumors $(55,56)$.

Today, immunotherapy for activating therapeutic anti-tumor immunity has become a mainstay of cancer therapy $(57,58)$. Although the use of monoclonal antibodies directed against cytotoxic T-lymphocyte associated protein 4 (CTLA-4) and the programmed cell death-1 (PD-1/CD279) T-cell receptor and/or its ligand (programmed death-ligand 1 (PD-L1/B7-H1/CD274) showed unprecedented durable responses in some patients with a variety of cancers, acquired resistance to immune checkpoint antibody blockades was commonly observed in most cancer patients $(59,60)$.

The different approaches being currently explored to increase recruitment of immune effector cells, include manipulating the expression of homing-associated molecules on T-cells and tumor endothelial cells. Concerning the first option, a successful approach to target or restore tumor-induced immunosuppression was made by adoptive cell therapy using tumor-reactive T-lymphocytes that resulted in objective tumor regression in $>50 \%$ of treated patients (61). The potential to treat a wide range of solid cancers with autologous T-cells was further highlighted when re-directed T-cells expressing a non-MHC restricted chimeric antibody receptor (recognizing CD19 on Bcells) in refractory B-cell malignancies were successfully used to overcome dominant immunosuppression $(62,63)$. However, the success of such therapies again depends on applied agents (here the lymphocytes) in finding their desired place, leaving the bloodstream and subsequently infiltrating the tumor tissues $(12,64)$.

Thus, strategies addressing directly the vascular system to sensitize tumors or improve the therapeutic response in cancer therapy were established and already shown to exert beneficial effects in immune checkpoint blockade. In a very elegant preclinical study Elia et al. showed that a selective (pre)activation of the tumor endothelium with the cytokine TNF promoted intratumoral T-cell infiltration, and immune checkpoint blockade (65). The authors used low doses of NGR-TNF, a Cys-Asn-Gly-Arg-Cys peptide-TNF fusion product, in simultaneous combination with anti-CTLA-4 and antiPD-1 antibodies to treat transgenic adenocarcinoma of the mouse prostate (TRAMP) mice with autochthonous prostate cancer and mice with orthotopic B16 melanoma. NGR-TNF administration was already used as a safe and therapeutic systemic administration to target TNF selectively to angiogenic tumor vessels which then altered the endothelial barrier function together with an upregulation of leukocyte-endothelial cell adhesion molecules, the release of pro-inflammatory cytokines, and the infiltration of tumor-specific effector CD8(+) T-cells. As a result, NGR-TNF enhanced the therapeutic activity of 
adoptive and active immunotherapy, delaying tumor growth and prolonging survival $(66,67)$. Finally, the combined therapy had beneficial effects on endogenous immune surveillance, through depletion of regulatory T-cells and expansion of a fully functional, polyclonal repertoire of cytotoxic T-lymphocytes (65).

Proper vascular function as revealed by measurements of vessel perfusion was further used to predict the therapeutic response to immune checkpoint blockade (68). Here, the authors used clinically relevant mouse breast tumor models that were either sensitive or resistant to immune checkpoint blockade treatment (with anti-CTLA4 and anti-PD1 agents) and thus mirror cancer progression and therapy response in humans. A significantly enhanced vessel perfusion was observed mostly in treatment-sensitive tumors, which was accompanied by an accumulation of CD8+ T-cells and interferon-gamma production, strongly suggested that increased vessel perfusion reflects the successful activation of anti-tumor T-cell immunity by immune checkpoint blockade (68). Thus, the authors reported here a reliable and noninvasive indicator for predicting immune checkpoint blockade responsiveness which was related to proper vascular function of the tumor endothelium.

Conclusively, tumor endothelial cells are actively involved in immune cell exclusion and inhibition of lymphocyte activation, fostering an immunosuppressive intratumoral microenvironment that contributes to the tumor immune escape and severely impairs conventional cancer therapies $(9,14,69)$. Hypothetically, tumors resistant to immune checkpoint blockade could become sensitive to such treatment again when the tumor endothelium specific alterations in leukocyte-endothelial adhesive interactions were normalized. In line with this idea, Huang et al. showed that synchronizing vascular normalization by antiangiogenic (anti-VEGFR2) therapy with T-cell activation induced by a whole cancer cell vaccine therapy enhanced anticancer efficacy in a CD8(+) T-cell-dependent manner in both immune-tolerant (MCaP0008) and immunogenic (MMTV-PyVT) murine breast cancer models (56). Even the administration of an antibody against mouse VEGF synergized with adoptive cell transfer-based immunotherapies (70). Herein, normalization of the tumor vasculature through disruption of the VEGF/VEGFR-2 axis increased extravasation of adoptively transferred T-cells into the tumor. Combining VEGF blockade with an additional blockade of angiopoietin-2 by a bispecific antibody provided superior therapeutic benefits in the melanoma cancer as well as in metastatic breast and pancreatic cancer models $(71,72)$. Neutralization of both angiogenic factors resulted in vascular regression of angiogenic blood vessels whereas the remaining blood vessels were normalized and facilitated the extravasation and perivascular accumulation of activated, IFN $\gamma$-expressing CD8(+) cytotoxic T lymphocytes (72). The perivascular T-cells in turn induced the expression of PD-L1 in tumor endothelial cells via IFN $\gamma$, which was utilized when additionally PD-1 blockade improved tumor control by the bispecific antibody in the different cancer models.

Using regulator of $G$ protein signaling 5-deficient mice, a genetically induced vascular normalization mouse model, in which newly formed blood vessels were characterized by a mature and thus stabilized phenotype, it was further shown that tumor vessel normalization consequently reduced vascular leakiness and hypoxia within the tumors, leading to an influx of immune effector T-cells $(22,30)$. Herein, vessel maturation was accompanied by a restoration of endothelial cell anergy as adhesion molecules on the luminal surface of tumor endothelial cells were increased and more uniformly distributed. Furthermore, the use of anti-angiogenic therapy was shown to normalize the tumor vasculature and thereby improve cancer immunotherapies.

Instead then of starving tumors from their blood supply and achieving complete vessel regression, vessel normalization by anti-angiogenic therapy has gained more attention for generating more mature and regular functioning tumor blood vessels with increased vessel perfusion. This is supposed to improve distribution of circulating blood components, oxygenation, removal of suppressive metabolites, as well as distribution of therapeutically applied drugs (56). In addition, anti-angiogenic therapy mediated vessel normalization was shown to reverse endothelial cell anergy resulting in (re)sensitizing tumor blood vessels to inflammatory stimuli by inducing homing molecule expression and thus an improved T-cell-dependent anti-cancer immunity $(12,70,73)$.

Improving the aberrant structural abnormalities and associated dysfunctionalities of tumor blood vessels, and thus lowering tumor hypoxia and enabling immune cell infiltration, by antiangiogenic therapy was shown to synergize with immunotherapies for more durable effects (74). In an preclinical study using the polyoma middle $\mathrm{T}$ oncoprotein breast cancer and the Rip1-Tag2 pancreatic neuroendocrine tumor mouse models it was shown that anti-angiogenic therapy can improve anti-PD-L1 treatment and further, the other way round that antiPD-L1 therapy can sensitize tumors to anti-angiogenic therapy and prolong its efficacy (74). Herein, vessel normalization (as shown by reduced microvessel densities, increased diameters and a regular pericyte coverage) promoted lymphocyte infiltration and enhanced cytotoxic T-cell activity.

In addition, to tumor endothelial cell anergy that limits the adhesion and subsequent extravasation of recruited leukocytes, tumor-derived factors can further induce endothelial cellmediated apoptosis of recruited immune cells, e.g., by induced death mediator Fas ligand (FasL, also called CD95L) expression which directly kills anti-tumor T-cells finally leading to an inefficient recruitment of effector CD8(+) T-cells into the tumor $(12,75)$. Within the tumor endothelium of breast, prostate, colon, bladder, renal cancers a selective expression of FasL was reported that was associated with scarce CD8(+) infiltration and a predominance of FoxP3(+) regulatory T-cells (76). As the induced FasL expression in tumor endothelial cells which acquired the ability to kill effector CD8(+) T-cells but not regulatory $\mathrm{T}$-cells was mediated by tumor-derived VEGF, IL10 and prostaglandin E2 cooperatively, the authors proposed a "tumor endothelial death barrier" that contributes to the tumors immune escape cells (76). The tumor endothelium was also shown to express increased levels of PD-L1 under inflammatory conditions, which in turn was able to bind to PD-1 on activated lymphocytes to negatively control T-cell activation (77-79). 
Another molecule which became of interest for activating therapeutic anti-tumor immunity is the interferon-inducible intracellular enzyme indoleamine 2,3-dioxygenase 1 (IDO1), which catalyzes the initial and rate-limiting step in the degradation pathway of the essential amino acid tryptophan to kynurenine $(80,81)$. Kynurenines in turn induces proliferation, activation and recruitment of $\mathrm{T}$ regulatory cells and myeloidderived suppressor cells that further suppress tumoricidal Tcells. Increased IDO-1 expression levels were already associated with tumor progression, poor prognosis, and a decreased overall survival $(82,83)$. IDO-1 expression can be found in different tumor cells, normal epithelial cells, monocyte-derived cells and in particular also in tumor endothelial cells (8486). Of note, in some tumor entities, the tumor endothelial cells rather than tumor cells were shown to be responsible for increased IDO expression, e.g., in metastatic renal cell carcinoma (84). IDO-1 expression levels in tumor endothelial cells were further suggested being a predictive biomarker for the response to immune-based cancer therapy (86-88). For example, in colorectal cancer, IDO-1 expression by host endothelial cells was a negative prognostic factor for regression free survival, independent of disease stage (89). Therefore, an inhibition of the (endothelial-specific) IDO-1 signaling pathway could be a promising novel adjuvant therapeutic strategy for clinical application in immunotherapy.

However, the actively participation of tumor endothelial cells in the innate and adaptive immune responses is not limited to the ability to attract and direct a wide range of immune cells and elevate extravasation from the host circulation. Tumor endothelial cells are believed to have a role in antigen presentation $(9,13,14)$. Endothelial cells were found to act as antigen presenting cells by constitutively expressing major histocompatibility complex I and II molecules and presenting endothelial antigens to $\mathrm{T}$-cells resulting in $\mathrm{T}$-cell activation $(90,91)$. Endothelial cells also were shown to express the costimulatory molecules CD80 and CD86 that are essential for activation of naïve T-cells, but following transplantation only activation of CD4(+) or CD8(+) T-cells was reported (92).

Conclusively these findings strongly argue for new therapeutic approaches including combinations of the anti-angiogenic treatments with immunotherapies in addition to the current standard regimens for cancers, particularly for those that do not respond to surgery, chemotherapy, or radiation.

\section{TUMOR ENDOTHELIUM MEDIATED IMMUNOLOGICAL CONSEQUENCES IN THE CONTEXT OF RADIOTHERAPY}

Tumor eradication or local cancer control for a better outcome are the main goals of radiation therapy. Endothelial cells act as critical determinants of the radiation response in tumors as radiotherapy generally fosters endothelial apoptosis, increased vascular permeability, and acquisition of a pro-inflammatory and -coagulant phenotype (93-95). The radiation sensitivity of vessels in general correlates with their morphology: capillaries and small vessels (like angiogenic tumor vessels) are extremely sensitive to ionizing radiation, whereas larger blood vessels seem to be less affected $(96,97)$. Radiation induces phenotypic changes of tumor endothelial cells (e.g., apoptosis or senescence) as well as wide range of microenvironmental changes by production and secretion of reactive oxygen and nitrogen species, growth and chemotactic factors, which in turn govern recruitment of immune cells $(11,98,99)$.

As an apparent approach, sensitizing tumor endothelial cells to radiation-induced apoptosis resulted in a more pronounced tumor growth delay upon irradiations in preclinical animal models, which suggested that a therapeutic targeting at the level of the tumor vasculature could counteract radiation resistance $(97,100)$. In contrast, in an elegant preclinical study Moding et al. reported that radiosensitizing endothelial cells did not increase local tumor control of soft tissue sarcomas after stereotactic body radiation therapy (101). Furthermore, proangiogenic factors including VEGF can rapidly repress radiation induced ceramide generation, and subsequently endothelial apoptosis (102). Therefore, targeting endothelial cells aiming at achieving complete tumor starvation is not supposed to be curative. More likely, approaches that improve the vascular function and thus tumor oxygenation as well as the recruitment and activation of immune cells by tumor endothelial cells gained attraction also in radiation therapy to enhance the sensitivity of the tumors to ionizing radiation.

To improve blood perfusion and thus tumor oxygenation, again vascular normalization using anti-angiogenic-therapy was suggested $(103,104)$. According to this hypothesis Koo et al. recently showed that a combined radiotherapy and anti-angiogenic treatment (with the second-generation multitargeted receptor tyrosine kinase inhibitor sunitinib malate, which inhibits PDGF and VEGF) showed synergistic effects in anti-cancer treatment using heterotopic human lung cancer xenografts (105). Herein, radiation induced extensive necrosis in the central portion of the tumors, as the immature tumor blood vessels were sensitive to radiotherapy. The resulting decreased vascular supply created then a hypoxic area and decreased the tumoricidal effect of radiotherapy by reducing the oxygen-free radicals. When radiotherapy was then combined with antiangiogenic treatment that inhibits the formation of immature blood vessels, the tumor perfusion was maintained and tumor necrosis was reduced. This treatment combination resulted then in a more significantly suppressed tumor growth, as vessel normalization, which achieved an efficient tumor perfusion, significantly improved tumor oxygenation that is prerequisite for the tumoricidal effects of ionizing radiation (105). In line with these findings Zhu et al. could show that inhibition of hypoxiainduced angiogenesis limits the efficiency of radiotherapy (106). Radiotherapy-sensitive lung tumors were characterized by low levels of hypoxia inducible factor- $1 \alpha$ and VEGF, which may reflect better oxygenated tumors with less angiogenic and thus more matured blood vessels. In contrast, high expression levels of the respective genes were detected in radiotherapy-resistant lung tumors which might be based on the hypoxic tumor microenvironment with more angiogenic tumor blood vessels (106). Conclusively, combining anti-angiogenic treatment with radiation therapy can achieve better tumor control as oxygen 
is a potent radiosensitizer; this may result in the use of lower radiation doses, as thus minimizing treatment-related normal tissue toxicity (107).

In general, the pharmacological inhibition of pro-angiogenic factors triggers apoptosis of angiogenic endothelial cells in the immature and leaky tumor blood vessels leading to the selection for mature, non-leaky vessels, the so-called pruning effect. Within these matured and normal vessels endothelial anergy is restored. Jaillet et al. reported that ionizing radiation altered the glycosylation pattern of endothelial cells, in particular increased high mannose-type $\mathrm{N}$-glycans and decreased glycosaminoglycans, which stimulated the interactions between irradiated endothelial cells and monocytes (108). Thus, targeting either the endothelium glycome may be considered as therapeutic target for modulating the inflammatory response or combining radiation therapy that seems to reduce endothelial anergy with anti-immunosuppressive therapy. Indeed, re-activation of the tumor vasculature was also shown to improve the therapeutic outcome of radiotherapy combined with immune-modulators. e.g., vessel specific-delivery of IL2, a cytokine known to stimulate the proliferation of cytotoxic T-cells, natural killer cells, and regulatory $\mathrm{T}$-cells, resulted in an additive or synergistic antitumor effect when the administration of this immunocytokine was combined with radiotherapy (109). Tumor endothelium specific targeting was achieved by coupling IL2 to the small immune protein L19 that recognizes the extra domain B (EDB) of fibronectin associated with tumor neovasculature. Of note, specifically addressing the tumor vasculature resulted in higher and thus more effective intratumoral local concentration of IL2 while reducing side effects, as the high doses used by systemic administration to reach an effective intratumoral dose of IL2 often leads to toxicity (e.g., capillary leakage) $(109,110)$.

In addition, preclinical and clinical evidence exists for the immuno-stimulatory properties of radiotherapy. Radiation treatment can foster immunogenic tumor cell death whereby danger-associated molecular patterns (DAMPs, e.g., calreticulin and adenosine triphosphate) were released which in turn can recruit and activate dendritic cells to process tumor antigens for naïve T-cells finally resulting in an anti-tumor immune responses $(111,112)$. Of note, radiation-induced tumor-targeted immunotherapy was shown to improve the therapeutic index and to extend the reach of immunomodulatory agents (113). In particular, radiation induced upregulation of VEGF expression was used to target $4-1 \mathrm{BB} / \mathrm{CD} 137$, a major immune-stimulatory receptor expressed on activated $\mathrm{CD} 8(+) \mathrm{T}$-cells, to the irradiated tumor as well as to distant tumor lesions. This innovative method used radiation therapy to extend tumor-targeted immunotherapy

\section{REFERENCES}

1. Folkman J. Tumor angiogenesis: a possible control point in tumor growth. Ann Intern Med. (1975) 82:96-100. doi: 10.7326/0003-4819-82-1-96

2. Carmeliet P, Jain RK. Angiogenesis in cancer and other diseases. Nature (2000) 407:249-57. doi: 10.1038/35025220 also to VEGF low tumors. Radiation-induced tissue injury, which is known to trigger angiogenic processes, is accompanied by upregulation of VEGF expression, especially in lesions expressing low levels of VEGF. The agonistic 4-1BB oligonucleotide aptamer was conjugated to an aptamer that binds to VEGF (114). The administration of this conjugate after tumor irradiation was used to induce an optimal $4-1 \mathrm{BB}$ co-stimulation at the tumor site that in turn enhanced tumor immunity and inhibited tumor growth, while no toxicities classically associated with systemic administration of 4-1BB ligands was observed. Thus, systemically administered but specifically tumor-/ VEGF-targeted 4-1BB costimulation in combination with radiation elicited a potent antitumor immune response capable of controlling the growth of distant non treated subcutaneous and metastatic breast tumor lesions (113). This anti-tumor T-cell activation as a result from tumor-localized radiation-induced anti-tumor immune responses strongly argues for a synergistic effect of radiotherapy with immune checkpoint inhibitors (115).

\section{CONCLUSION}

The tumor vascular endothelium is a key cell compartment for the response of tumors to cancer therapy. The tumor initiated neovascularization for nutrients and oxygen supply prior tumor progression results in a structural and functional abnormal tumor vasculature, which contributes to a pro-tumorigenic and immunosuppressive environment altering the therapy response of tumor cells. In particular for clinically approved immunotherapies, such as immune checkpoint blockade and adoptive T-cell transfer, the functional abnormal tumor vasculature fosters therapy resistance by limiting an inefficient recruitment, distribution and infiltration of tumor eradicating immune cells. Therefore, tumor vasculature targeting agents in order to re-activate specifically the tumor endothelial cells in terms of vascular normalization provide promising strategies to optimize the efficacy of currently employed cancer therapies, especially immunotherapies.

\section{AUTHOR CONTRIBUTIONS}

The author confirms being the sole contributor of this work and approved it for publication.

\section{FUNDING}

The work was supported by grants of the DFG (GRK1739/2), the BMBF (ZISS 02NUK024-D), and the Brigitte und Dr. Konstanze Wegener-Stiftung. 
Cell (1996) 86:353-64. doi: $\quad 10.1016 /$ S0092-8674(00)80

108-7

6. Bergers G, Benjamin LE. Tumorigenesis and the angiogenic switch. Nat Rev Cancer (2003) 3:401-10. doi: 10.1038/nrc1093

7. Tilki D, Kilic N, Sevinc S, Zywietz F, Stief CG, Ergun S. Zone-specific remodeling of tumor blood vessels affects tumor growth. Cancer (2007) 110:2347-62. doi: 10.1002/cncr.23024

8. Gaengel K, Genove G, Armulik A, Betsholtz C. Endothelial-mural cell signaling in vascular development and angiogenesis. Arterioscler Thromb Vasc Biol. (2009) 29:630-8. doi: 10.1161/ATVBAHA.107.161521

9. Mai J, Virtue A, Shen J, Wang H, Yang XF. An evolving new paradigm: endothelial cells-conditional innate immune cells. J Hematol Oncol. (2013) 6:61. doi: 10.1186/1756-8722-6-61

10. Klein D. Vascular wall-resident multipotent stem cells of mesenchymal nature within the process of vascular remodeling: cellular basis, clinical relevance, and implications for stem cell therapy. Stem Cells Int. (2016) 2016:1905846. doi: 10.1155/2016/1905846

11. Guipaud O, Jaillet C, Clement-Colmou K, Francois A, Supiot S, Milliat F. The importance of the vascular endothelial barrier in the immuneinflammatory response induced by radiotherapy. Br J Radiol. (2018). doi: 10.1259/bjr.20170762. [Epub ahead of print].

12. Ager A, Watson HA, Wehenkel SC, Mohammed RN. Homing to solid cancers: a vascular checkpoint in adoptive cell therapy using CAR T-cells. Biochem Soc Trans. (2016) 44:377-85. doi: 10.1042/BST20150254

13. Al-Soudi A, Kaaij MH, Tas SW. Endothelial cells: from innocent bystanders to active participants in immune responses. Autoimmun Rev. (2017) 16:95162. doi: 10.1016/j.autrev.2017.07.008

14. De Sanctis F, Ugel S, Facciponte J, Facciabene A. The dark side of tumor-associated endothelial cells. Semin Immunol. (2018) 35:35-47. doi: 10.1016/j.smim.2018.02.002

15. Ribatti D, Nico B, Crivellato E, Vacca A. The structure of the vascular network of tumors. Cancer Lett. (2007) 248:18-23. doi: 10.1016/j.canlet.2006.06.007

16. Weisshardt P, Trarbach T, Durig J, Paul A, Reis H, Tilki D, et al. Tumor vessel stabilization and remodeling by anti-angiogenic therapy with bevacizumab. Histochem Cell Biol. (2012) 137:391-401. doi: 10.1007/s00418-0110898-8

17. Tilki D, Seitz M, Singer BB, Irmak S, Stief CG, Reich O, et al. Molecular imaging of tumor blood vessels in prostate cancer. Anticancer Res. (2009) 29:1823-9. Available online at: http://ar.iiarjournals.org/content/29/5/1823. long

18. Jain RK. Antiangiogenesis strategies revisited: from starving tumors to alleviating hypoxia. Cancer Cell (2014) 26:605-22. doi: 10.1016/j.ccell.2014.10.006

19. Wang Z, Dabrosin C, Yin X, Fuster MM, Arreola A, Rathmell WK., et al. Broad targeting of angiogenesis for cancer prevention and therapy. Semin Cancer Biol. (2015) 35(Suppl.):S224-43. doi: 10.1016/j.semcancer.2015.01.001

20. Ye W. The complexity of translating anti-angiogenesis therapy from basic science to the clinic. Dev Cell (2016) 37:114-25. doi: 10.1016/j.devcel.2016.03.015

21. Steyers CM III, Miller FJ Jr. Endothelial dysfunction in chronic inflammatory diseases. Int J Mol Sci. (2014) 15:11324-49. doi: 10.3390/ijms150711324

22. Jung K, Heishi T, Khan OF, Kowalski PS, Incio J, Rahbari NN, et al. Ly6Clo monocytes drive immunosuppression and confer resistance to anti-VEGFR2 cancer therapy. J Clin Invest. (2017) 127:3039-51. doi: 10.1172/JCI93182

23. Willms-Kretschmer K, Flax MH, Cotran RS. The fine structure of the vascular response in hapten-specific delayed hypersensitivity and contact dermatitis. Lab Invest. (1967) 17:334-49.

24. Pober JS. Warner-Lambert/Parke-Davis award lecture. Cytokine-mediated activation of vascular endothelium physiology and pathology. Am J Pathol. (1988) 133:426-33.

25. Hunt BJ, Jurd KM. Endothelial cell activation. A central pathophysiological process. BMJ (1998) 316:1328-9. doi: 10.1136/bmj.316.7141.1328

26. Lee WL, Liles WC. Endothelial activation, dysfunction and permeability during severe infections. Curr Opin Hematol. (2011) 18:191-6. doi: 10.1097/MOH.0b013e328345a3d1
27. Cotran RS, Pober JS. Effects of cytokines on vascular endothelium: their role in vascular and immune injury. Kidney Int. (1989) 35:969-75. doi: $10.1038 /$ ki.1989.80

28. Leung DY, Cotran RS, Kurt-Jones E, Burns JC, Newburger JW, Pober JS. Endothelial cell activation and high interleukin-1 secretion in the pathogenesis of acute Kawasaki disease. Lancet (1989) 2:1298-302. doi: 10.1016/S0140-6736(89)91910-7

29. Bach FH, Hancock WW, Ferran C. Protective genes expressed in endothelial cells: a regulatory response to injury. Immunol Today (1997) 18:483-6. doi: 10.1016/S0167-5699(97)01129-8

30. Rahbari NN, Kedrin D, Incio J, Liu H, Ho WW, Nia HT, et al. AntiVEGF therapy induces ECM remodeling and mechanical barriers to therapy in colorectal cancer liver metastases. Sci Transl Med. (2016) 8:360ra135. doi: $10.1126 /$ scitranslmed.aaf5219

31. Liao JK. Linking endothelial dysfunction with endothelial cell activation. $J$ Clin Invest. (2013) 123:540-1. doi: 10.1172/JCI66843

32. Dudley AC. Tumor endothelial cells. Cold Spring Harb Perspect Med. (2012) 2:a006536. doi: 10.1101/cshperspect.a006536

33. Wilhelm K, Happel K, Eelen G, Schoors S, Oellerich MF, Lim R, et al. FOXO1 couples metabolic activity and growth state in the vascular endothelium. Nature (2016) 529:216-20. doi: 10.1038/nature16498

34. Hida K, Maishi N, Annan DA, Hida Y. Contribution of tumor endothelial cells in cancer progression. Int J Mol Sci. (2018) 19:E1272. doi: 10.3390/ijms19051272

35. Hida K, Hida Y, Amin DN, Flint AF, Panigrahy D, Morton CC, et al. Tumor-associated endothelial cells with cytogenetic abnormalities. Cancer Res. (2004) 64:8249-55. doi: 10.1158/0008-5472.CAN-04-1567

36. Akino T, Hida K, Hida Y, Tsuchiya K, Freedman D, Muraki C, et al. Cytogenetic abnormalities of tumor-associated endothelial cells in human malignant tumors. Am J Pathol. (2009) 175:2657-67. doi: 10.2353/ajpath.2009.090202

37. Jain RK. Normalization of tumor vasculature: an emerging concept in antiangiogenic therapy. Science (2005) 307:58-62. doi: $10.1126 /$ science. 1104819

38. Goel S, Duda DG, Xu L, Munn LL, Boucher Y, Fukumura D, et al. Normalization of the vasculature for treatment of cancer and other diseases. Physiol Rev. (2011) 91:1071-121. doi: 10.1152/physrev.00038.2010

39. Baxter LT, Jain RK. Transport of fluid and macromolecules in tumors. II Role of heterogeneous perfusion and lymphatics. Microvasc Res. (1990) 40:246-63. doi: 10.1016/0026-2862(90)90023-K

40. Boucher Y, Baxter LT, Jain RK. Interstitial pressure gradients in tissueisolated and subcutaneous tumors: implications for therapy. Cancer Res. (1990) 50:4478-84.

41. Ohga N, Ishikawa S, Maishi N, Akiyama K, Hida Y, Kawamoto T, et al. Heterogeneity of tumor endothelial cells: comparison between tumor endothelial cells isolated from high- and low-metastatic tumors. Am J Pathol. (2012) 180:1294-307. doi: 10.1016/j.ajpath.2011.11.035

42. Cairns R, Papandreou I, Denko N. Overcoming physiologic barriers to cancer treatment by molecularly targeting the tumor microenvironment. Mol Cancer Res. (2006) 4:61-70. doi: 10.1158/1541-7786.MCR-06-0002

43. Yeo SG, Kim JS, Cho MJ, Kim KH. Interstitial fluid pressure as a prognostic factor in cervical cancer following radiation therapy. Clin Cancer Res. (2009) 15:6201-7. doi: 10.1158/1078-0432.CCR-09-0560

44. Rofstad EK, Galappathi K, Mathiesen BS. Tumor interstitial fluid pressure-a link between tumor hypoxia, microvascular density, and lymph node metastasis. Neoplasia (2014) 16:586-94. doi: 10.1016/j.neo. 2014.07.003

45. Piali L, Fichtel A, Terpe HJ, Imhof BA, Gisler RH. Endothelial vascular cell adhesion molecule 1 expression is suppressed by melanoma and carcinoma. J Exp Med. (1995) 181:811-6. doi: 10.1084/jem.181.2.811

46. Griffioen AW, Damen CA, Blijham GH, Groenewegen G. Tumor angiogenesis is accompanied by a decreased inflammatory response of tumor-associated endothelium. Blood (1996) 88:667-73.

47. Griffioen AW, Damen CA, Martinotti S, Blijham GH, Groenewegen G. Endothelial intercellular adhesion molecule-1 expression is suppressed in human malignancies: the role of angiogenic factors. Cancer Res. (1996) 56:1111-7. 
48. Griffioen AW, Damen CA, Mayo KH, Barendsz-Janson AF, Martinotti $\mathrm{S}$, Blijham $\mathrm{GH}$, et al. Angiogenesis inhibitors overcome tumor induced endothelial cell anergy. Int J Cancer (1999) 80:315-9. doi: 10.1002/(SICI)1097-0215(19990118)80:2<315::AID-IJC23>3.0.CO;2-L

49. Rosenberg SA, Restifo NP, Yang JC, Morgan RA, Dudley ME. Adoptive cell transfer: a clinical path to effective cancer immunotherapy. Nat Rev Cancer (2008) 8:299-308. doi: 10.1038/nrc2355

50. Dougan M, Dranoff G. Immune therapy for cancer. Annu Rev Immunol. (2009) 27:83-117. doi: 10.1146/annurev.immunol.021908.132544

51. Betsholtz C. Vascular biology: transcriptional control of endothelial energy. Nature (2016) 529:160-1. doi: 10.1038/nature16866

52. Flati V, Pastore LI, Griffioen AW, Satijn S, Toniato E, D'alimonte I, et al. Endothelial cell anergy is mediated by bFGF through the sustained activation of p38-MAPK and NF-kappaB inhibition. Int J Immunopathol Pharmacol. (2006) 19:761-73. doi: 10.1177/039463200601900406

53. Hellebrekers DM, Castermans K, Vire E, Dings RP, Hoebers NT, Mayo $\mathrm{KH}$, et al. Epigenetic regulation of tumor endothelial cell anergy: silencing of intercellular adhesion molecule-1 by histone modifications. Cancer Res. (2006) 66:10770-7. doi: 10.1158/0008-5472.CAN-06-1609

54. Taylor CT, Colgan SP. Regulation of immunity and inflammation by hypoxia in immunological niches. Nat Rev Immunol. (2017) 17:774-85. doi: 10.1038/nri.2017.103

55. Huang Y, Kim BYS, Chan CK, Hahn SM, Weissman IL, Jiang W. Improving immune-vascular crosstalk for cancer immunotherapy. Nat Rev Immunol. (2018) 18:195-203. doi: 10.1038/nri.2017.145

56. Huang Y, Yuan J, Righi E, Kamoun WS, Ancukiewicz M, Nezivar $J$, et al. Vascular normalizing doses of antiangiogenic treatment reprogram the immunosuppressive tumor microenvironment and enhance immunotherapy. Proc Natl Acad Sci USA. (2012) 109:17561-6. doi: $10.1073 /$ pnas. 1215397109

57. Pardoll DM. The blockade of immune checkpoints in cancer immunotherapy. Nat Rev Cancer (2012) 12:252-64. doi: 10.1038/nrc3239

58. Li X, Shao C, Shi Y, Han W. Lessons learned from the blockade of immune checkpoints in cancer immunotherapy. J Hematol Oncol. (2018) 11:31. doi: 10.1186/s13045-018-0578-4

59. Tang H, Wang Y, Chlewicki LK, Zhang Y, Guo J, Liang W, et al. Facilitating T Cell infiltration in tumor microenvironment overcomes resistance to PD-L1 blockade. Cancer Cell (2016) 29:285-96. doi: 10.1016/j.ccell.2016.02.004

60. Sharma P, Hu-Lieskovan S, Wargo JA, Ribas A. Primary, adaptive, and acquired resistance to cancer immunotherapy. Cell (2017) 168:707-23. doi: 10.1016/j.cell.2017.01.017

61. Dudley ME, Wunderlich JR, Robbins PF, Yang JC, Hwu P, Schwartzentruber DJ, et al. Cancer regression and autoimmunity in patients after clonal repopulation with antitumor lymphocytes. Science (2002) 298:850-4. doi: 10.1126/science.1076514

62. Brentjens RJ, Riviere I, Park JH, Davila ML, Wang X, Stefanski J, et al. Safety and persistence of adoptively transferred autologous CD19-targeted T cells in patients with relapsed or chemotherapy refractory B-cell leukemias. Blood (2011) 118:4817-28. doi: 10.1182/blood-2011-04-348540

63. Brentjens RJ, Davila ML, Riviere I, Park J, Wang X, Cowell LG, et al. CD19-targeted $\mathrm{T}$ cells rapidly induce molecular remissions in adults with chemotherapy-refractory acute lymphoblastic leukemia. Sci Transl Med. (2013) 5:177ra138. doi: 10.1126/scitranslmed.3005930

64. Nishino M, Ramaiya NH, Hatabu H, Hodi FS. Monitoring immunecheckpoint blockade: response evaluation and biomarker development. Nat Rev Clin Oncol. (2017) 14:655-68. doi: 10.1038/nrclinonc.2017.88

65. Elia AR, Grioni M, Basso V, Curnis F, Freschi M, Corti A, et al. Targeting tumor vasculature with TNF leads effector T Cells to the tumor and enhances therapeutic efficacy of immune checkpoint blockers in combination with adoptive cell therapy. Clin Cancer Res. (2018) 24:2171-81. doi: 10.1158/1078-0432.CCR-17-2210

66. Corti A, Curnis F, Arap W, Pasqualini R. The neovasculature homing motif NGR: more than meets the eye. Blood (2008) 112:2628-35. doi: 10.1182/blood-2008-04-150862

67. Calcinotto A, Grioni M, Jachetti E, Curnis F, Mondino A, Parmiani $\mathrm{G}$, et al. Targeting TNF-alpha to neoangiogenic vessels enhances lymphocyte infiltration in tumors and increases the therapeutic potential of immunotherapy. J Immunol. (2012) 188:2687-94 doi: 10.4049/jimmunol.1101877

68. Zheng X, Fang Z, Liu X, Deng S, Zhou P, Wang X, et al. Increased vessel perfusion predicts the efficacy of immune checkpoint blockade. J Clin Invest. (2018) 128:2104-15. doi: 10.1172/JCI96582

69. Kazerounian S, Lawler J. Integration of pro- and anti-angiogenic signals by endothelial cells. J Cell Commun Signal. (2018) 12:171-9. doi: 10.1007/s12079-017-0433-3

70. Shrimali RK, Yu Z, Theoret MR, Chinnasamy D, Restifo NP, Rosenberg SA. Antiangiogenic agents can increase lymphocyte infiltration into tumor and enhance the effectiveness of adoptive immunotherapy of cancer. Cancer Res. (2010) 70:6171-80. doi: 10.1158/0008-5472.CAN-10-0153

71. Schmittnaegel $M$, De Palma $M$. reprogramming tumor blood vessels for enhancing immunotherapy. Trends Cancer (2017) 3:809-12. doi: 10.1016/j.trecan.2017.10.002

72. Schmittnaegel M, Rigamonti N, Kadioglu E, Cassara A, Wyser Rmili C, Kiialainen A, et al. Dual angiopoietin-2 and VEGFA inhibition elicits antitumor immunity that is enhanced by PD-1 checkpoint blockade. Sci Transl Med. (2017) 9:eaak9670. doi: 10.1126/scitranslmed.aak9670

73. Hamzah J, Jugold M, Kiessling F, Rigby P, Manzur M, Marti HH, et al. Vascular normalization in Rgs5-deficient tumours promotes immune destruction. Nature (2008) 453:410-4. doi: 10.1038/nature06868

74. Allen E, Jabouille A, Rivera LB, Lodewijckx I, Missiaen R, Steri V, et al. Combined antiangiogenic and anti-PD-L1 therapy stimulates tumor immunity through HEV formation. Sci Transl Med. (2017) 9:eaak9679. doi: 10.1126/scitranslmed.aak9679

75. Joyce JA, Fearon DT. T cell exclusion, immune privilege, and the tumor microenvironment. Science (2015) 348:74-80. doi: 10.1126/science.aaa6204

76. Motz GT, Santoro SP, Wang LP, Garrabrant T, Lastra RR, Hagemann IS, et al. Tumor endothelium FasL establishes a selective immune barrier promoting tolerance in tumors. Nat Med. (2014) 20:607-15. doi: 10.1038/nm.3541

77. Bichsel CA, Wang L, Froment L, Berezowska S, Muller S, Dorn $\mathrm{P}$, et al. Increased PD-L1 expression and IL-6 secretion characterize human lung tumor-derived perivascular-like cells that promote vascular leakage in a perfusable microvasculature model. Sci Rep. (2017) 7:10636. doi: 10.1038/s41598-017-09928-1

78. Bagaria SP, Gatalica Z, Maney T, Serie D, Parasramka M, Attia S, et al. Association between programmed death-ligand 1 expression and the vascular endothelial growth factor pathway in angiosarcoma. Front Oncol. (2018) 8:71. doi: 10.3389/fonc.2018.00071

79. Jennewein L, Bartsch G, Gust K, Kvasnicka HM, Haferkamp A, Blaheta R, et al. Increased tumor vascularization is associated with the amount of immune competent PD-1 positive cells in testicular germ cell tumors. Oncol Lett. (2018) 15:9852-60. doi: 10.3892/ol.2018.8597

80. Holmgaard RB, Zamarin D, Li Y, Gasmi B, Munn DH, Allison JP, et al. Tumor-expressed IDO recruits and activates MDSCs in a TREG-dependent manner. Cell Rep. (2015) 13:412-24. doi: 10.1016/j.celrep.2015.08.077

81. Wu H, Gong J, Liu Y. Indoleamine 2, 3-dioxygenase regulation of immune response (Review). Mol Med Rep. (2018) 17:4867-73. doi: 10.3892/mmr.2018.8537

82. Moon YW, Hajjar J, Hwu P, Naing A. Targeting the indoleamine 2,3-dioxygenase pathway in cancer. J Immunother Cancer (2015) 3:51. doi: 10.1186/s40425-015-0094-9

83. Brochez L, Chevolet I, Kruse V. The rationale of indoleamine 2,3dioxygenase inhibition for cancer therapy. Eur J Cancer (2017) 76:167-82. doi: 10.1016/j.ejca.2017.01.011

84. Riesenberg R, Weiler C, Spring O, Eder M, Buchner A, Popp T, et al. Expression of indoleamine 2,3-dioxygenase in tumor endothelial cells correlates with long-term survival of patients with renal cell carcinoma. Clin Cancer Res. (2007) 13:6993-7002. doi: 10.1158/1078-0432.CCR-07-0942

85. Munn DH, Mellor AL. IDO in the tumor microenvironment: inflammation, counter-regulation, and tolerance. Trends Immunol. (2016) 37:193-207. doi: 10.1016/j.it.2016.01.002

86. Seeber A, Klinglmair G, Fritz J, Steinkohl F, Zimmer KC, Aigner F, et al. High IDO-1 expression in tumor endothelial cells is associated with response to immunotherapy in metastatic renal cell carcinoma. Cancer Sci. (2018) 109:1583-91. doi: 10.1111/cas.13560 
87. Chevolet I, Speeckaert R, Haspeslagh M, Neyns B, Kruse V, Schreuer M, et al. Peritumoral indoleamine 2,3-dioxygenase expression in melanoma: an early marker of resistance to immune control? Br J Dermatol. (2014) 171:987-95. doi: 10.1111/bjd.13100

88. Krahenbuhl L, Goldinger SM, Mangana J, Kerl K, Chevolet I, Brochez L, et al. A longitudinal analysis of IDO and PDL1 expression during immuneor targeted therapy in advanced melanoma. Neoplasia (2018) 20:218-25. doi: 10.1016/j.neo.2017.12.002

89. Meireson A, Chevolet I, Hulstaert E, Ferdinande L, Ost P, Geboes K, et al. Peritumoral endothelial indoleamine 2, 3-dioxygenase expression is an early independent marker of disease relapse in colorectal cancer and is influenced by DNA mismatch repair profile. Oncotarget (2018) 9:25216-24. doi: 10.18632 /oncotarget.25393

90. Wheway J, Latham SL, Combes V, Grau GE. Endothelial microparticles interact with and support the proliferation of T cells. J Immunol. (2014) 193:3378-87. doi: 10.4049/jimmunol.1303431

91. Carman CV, Martinelli R. T lymphocyte-endothelial interactions: emerging understanding of trafficking and antigen-specific immunity. Front Immunol. (2015) 6:603. doi: 10.3389/fimmu.2015.00603

92. Pober JS, Tellides G. Participation of blood vessel cells in human adaptive immune responses. Trends Immunol. (2012) 33:49-57. doi: 10.1016/j.it.2011.09.006

93. Baker DG, Krochak RJ. The response of the microvascular system to radiation: a review. Cancer Invest. (1989) 7:287-94. doi: $10.3109 / 07357908909039849$

94. Paris F, Fuks Z, Kang A, Capodieci P, Juan G, Ehleiter D, et al. Endothelial apoptosis as the primary lesion initiating intestinal radiation damage in mice. Science (2001) 293:293-7. doi: 10.1126/science.1060191

95. Rannou E, Francois A, Toullec A, Guipaud O, Buard V, Tarlet G, et al. In vivo evidence for an endothelium-dependent mechanism in radiation-induced normal tissue injury. Sci Rep. (2015) 5:15738. doi: 10.1038/srep15738

96. Fajardo LF. The pathology of ionizing radiation as defined by morphologic patterns. Acta Oncol. (2005) 44:13-22. doi: 10.1080/02841860510007440

97. Klein D, Schmitz T, Verhelst V, Panic A, Schenck M, Reis H, et al. Endothelial Caveolin-1 regulates the radiation response of epithelial prostate tumors. Oncogenesis (2015) 4:e148. doi: 10.1038/oncsis.2015.9

98. Oh ET, Park MT, Song MJ, Lee H, Cho YU, Kim SJ, et al. Radiationinduced angiogenic signaling pathway in endothelial cells obtained from normal and cancer tissue of human breast. Oncogene (2014) 33:1229-38. doi: $10.1038 /$ onc. 2013.70

99. Wang Y, Boerma M, Zhou D. Ionizing radiation-induced endothelial cell senescence and cardiovascular diseases. Radiat Res. (2016) 186:153-61. doi: $10.1667 /$ RR14445.1

100. Tavora B, Reynolds LE, Batista S, Demircioglu F, Fernandez I, Lechertier T, et al. Endothelial-cell FAK targeting sensitizes tumours to DNA-damaging therapy. Nature (2014) 514:112-6. doi: 10.1038/nature13541

101. Moding EJ, Castle KD, Perez BA, Oh P, Min HD, Norris H, et al. (2015). Tumor cells, but not endothelial cells, mediate eradication of primary sarcomas by stereotactic body radiation therapy. Sci Transl Med. 7:278ra234. doi: 10.1126/scitranslmed.aaa4214

102. Leroi N, Lallemand F, Coucke P, Noel A, Martinive P. Impacts of ionizing radiation on the different compartments of the tumor microenvironment. Front Pharmacol. (2016) 7:78. doi: 10.3389/fphar.2016.00078

103. Sorensen AG, Emblem KE, Polaskova P, Jennings D, Kim H, Ancukiewicz $\mathrm{M}$, et al. Increased survival of glioblastoma patients who respond to antiangiogenic therapy with elevated blood perfusion. Cancer Res. (2012) 72:402-7. doi: 10.1158/0008-5472.CAN-11-2464
104. Yoo DS, Kirkpatrick JP, Craciunescu O, Broadwater G, Peterson BL, Carroll $\mathrm{MD}$, et al. Prospective trial of synchronous bevacizumab, erlotinib, and concurrent chemoradiation in locally advanced head and neck cancer. Clin Cancer Res. (2012) 18:1404-14. doi: 10.1158/1078-0432.CCR-11-1982

105. Koo HJ, Lee M, Kim J, Woo CW, Jeong SY, Choi EK, et al. Synergistic effect of anti-angiogenic and radiation therapy: quantitative evaluation with dynamic contrast enhanced MR imaging. PLoS ONE (2016) 11:e0148784. doi: 10.1371 /journal.pone. 0148784

106. Zhu H, Zhang S. Hypoxia inducible factor- $1 \alpha /$ vascular endothelial growth factor signaling activation correlates with response to radiotherapy and its inhibition reduces hypoxia-induced angiogenesis in lung cancer. J Cell Biochem. (2018). doi: 10.1002/jcb.27120. [Epub ahead of print].

107. Wang $\mathrm{H}, \mathrm{MuX}, \mathrm{He} \mathrm{H}$, Zhang XD. Cancer radiosensitizers. Trends Pharmacol Sci. (2018) 39:24-48. doi: 10.1016/j.tips.2017.11.003

108. Jaillet C, Morelle W, Slomianny MC, Paget V, Tarlet G, Buard V, et al. Radiation-induced changes in the glycome of endothelial cells with functional consequences. Sci Rep. (2017) 7:5290. doi: 10.1038/s41598-017-05563-y

109. Zegers CM, Rekers NH, Quaden DH, Lieuwes NG, Yaromina A, Germeraad WT, et al. Radiotherapy combined with the immunocytokine L19-IL2 provides long-lasting antitumor effects. Clin Cancer Res. (2015) 21:1151-60. doi: 10.1158/1078-0432.CCR-14-2676

110. Alwan LM, Grossmann K, Sageser D, Van Atta J, Agarwal N, Gilreath JA. Comparison of acute toxicity and mortality after two different dosing regimens of high-dose interleukin-2 for patients with metastatic melanoma. Target Oncol. (2014) 9:63-71. doi: 10.1007/s11523-013-0276-7

111. Golden EB, Frances D, Pellicciotta I, Demaria S, Helen Barcellos-Hoff M, Formenti SC. Radiation fosters dose-dependent and chemotherapyinduced immunogenic cell death. Oncoimmunology (2014) 3:e28518. doi: $10.4161 /$ onci.28518

112. Frey B, Hehlgans S, Rodel F, Gaipl US. Modulation of inflammation by low and high doses of ionizing radiation: implications for benign and malign diseases. Cancer Lett. (2015) 368:230-7. doi: 10.1016/j.canlet.2015. 04.010

113. Schrand B, Verma B, Levay A, Patel S, Castro I, Benaduce AP, et al. Radiation-induced enhancement of antitumor T-cell immunity by VEGF-targeted 4-1BB costimulation. Cancer Res. (2017) 77:1310-21. doi: 10.1158/0008-5472.CAN-16-2105

114. Schrand B, Berezhnoy A, Brenneman R, Williams A, Levay A, Kong LY, et al. Targeting 4-1BB costimulation to the tumor stroma with bispecific aptamer conjugates enhances the therapeutic index of tumor immunotherapy. Cancer Immunol Res. (2014) 2:867-77. doi: 10.1158/2326-6066.CIR-14-0007

115. Wennerberg E, Lhuillier C, Vanpouille-Box C, Pilones KA, GarciaMartinez E, Rudqvist NP, et al. Barriers to radiation-induced in situ tumor vaccination. Front Immunol. (2017) 8:229. doi: 10.3389/fimmu.2017. 00229

Conflict of Interest Statement: The author declares that the research was conducted in the absence of any commercial or financial relationships that could be construed as a potential conflict of interest.

Copyright $\odot 2018$ Klein. This is an open-access article distributed under the terms of the Creative Commons Attribution License (CC BY). The use, distribution or reproduction in other forums is permitted, provided the original author(s) and the copyright owner(s) are credited and that the original publication in this journal is cited, in accordance with accepted academic practice. No use, distribution or reproduction is permitted which does not comply with these terms. 